\title{
Jogo Sério para reabilitação do equilíbrio de hemiparéticos por acidente vascular cerebral
}

\author{
Serious game for balance rehabilitation of \\ hemiparetic stroke patients
}

\section{Juego serio para la rehabilitación del equilibrio de hemiparéticos debido a accidente cerebrovascular}

\author{
Rafaela Korn ${ }^{1}$, Danielle Fernandes dos Santos ${ }^{2}$, Antonio Vinicius \\ Soares $^{3}$, Fabrício Noveletto ${ }^{4}$, Fernando Luís Fischer Eichinger ${ }^{5}$ \\ 1.Fisioterapeuta, Faculdade Guilherme Guimbala, Joinville-SC, Brasil. Orcid https://orcid.org/0000-0002- \\ 0243-5134 \\ 2.Fisioterapeuta, Faculdade Guilherme Guimbala, Joinville-SC, Brasil. Orcid https://orcid.org/0000-0003- \\ $1212-429 \mathrm{X}$ \\ 3.Fisioterapeuta, Doutor em Ciências do Movimento Humano. Professor da Universidade de Região de \\ Joinville - UNIVILLE e da Faculdade IELUSC, Joinville-SC, Brasil. Orcid https://orcid.org/0000-0001-6090- \\ $\underline{1423}$ \\ 4.Engenheiro Elétrico, Doutor em Engenharia Elétrica. Professor da Universidade do Estado de Santa \\ Catarina - CCT/UDESC, Joinville-SC, Brasil. Orcid https://orcid.org/0000-0003-0310-4262 \\ 5.Fisioterapeuta, Mestre em Ciências do Movimento Humano. Professor da Faculdade Guilherme Guimbala \\ e da Universidade da Região de Joinville, Joinville-SC, Brasil. Orcid https://orcid.org/0000-0002-4385- \\ $\underline{4853}$
}

\section{Resumo}

Introdução. Pacientes hemiparéticos por acidente vascular cerebral (AVC) possuem déficits funcionais residuais, incluindo alterações do equilíbrio, o que impacta na qualidade de vida. 0 uso de tecnologias inovadoras tem aumentado na reabilitação, entre estes recursos estão os Jogos Sérios (JS). Objetivo. Verificar os efeitos terapêuticos de um programa de exercícios utilizando um JS desenvolvido para avaliação e reabilitação do equilíbrio de pacientes hemiparéticos por AVC. Método. Trata-se de um estudo quase-experimental do tipo séries de tempo, envolvendo 12 pacientes com hemiparesia pós-AVC. A intervenção durou 10 semanas, com as sessões realizadas duas vezes por semana. Foram avaliados: força muscular de quadríceps femoral (dinamometria), equilíbrio (estabilometria dinâmica e Escala de Equilíbrio de Berg), mobilidade funcional (Timed Up and Go Test) e percepção da qualidade de vida (Perfil de Saúde de Nottingham). Resultados. Melhoras significativas e com grande tamanho de efeito (TDE) foram observadas em todas as variáveis, com exceção da força muscular no membro não parético, que apresentou TDE moderado. Destacam-se os incrementos em todos os testes de equilíbrio ( $p<0,001 ;$ TDE: 0,9$)$. Conclusões. Um programa de exercícios com JS parece ser uma boa opção de tratamento para pacientes hemiparéticos após AVC, especialmente para a melhora do equilíbrio. Este recurso pode contribuir no processo de reabilitação destes pacientes.

Unitermos. Jogos de Vídeo; Reabilitação; Equilíbrio Postural; Hemiparesia; Acidente Vascular Cerebral

\footnotetext{
Abstract

Introduction. Hemiparetic stroke patients have residual functional deficits, including changes in balance, which impacts on quality of life. The use of innovative technologies has increased, among these resources are the Serious Games (SG). Objective. To verify the therapeutic effects of an exercise program using a SG developed for assessment and rehabilitation of the balance of hemiparetic post-stroke. Method. This is a quasi-experimental time series study involving 12 patients with post-stroke hemiparesis. The intervention lasted 10 weeks, twice a week. Quadriceps femoris muscle strength (dynamometry), balance (dynamic stabilometry
} 
and Berg Balance Scale), functional mobility (Timed Up and Go Test) and perception of quality of life (Nottingham Health Profile) were evaluated. Results. Significant improvements with large effect size (ES) were observed in all variables except muscle strength in the non-paretic limb, which showed moderate ES. Increments in all balance tests should be highlighted $(p<0.001 ;$ ES: 0.9). Conclusions. An exercise program using a SG seems to be a good treatment option for hemiparetic patients after stroke, especially for balance improvement. This feature can contribute to the rehabilitation process of these patients.

Keywords. Video Games; Rehabilitation; Postural Balance; Hemiparesis; Stroke

\begin{abstract}
Resumen
Introducción. Los hemiparéticos debido a un accidente cerebrovascular (ACV) tienen déficits funcionales residuales, incluido el equilibrio alterado, que repercute en la calidad de vida. Se ha incrementado el uso de tecnologías innovadoras en rehabilitación, entre estos recursos se encuentran los Juegos Serios (JS). Objetivo. Verificar los efectos terapéuticos de un programa de ejercicios utilizando un JS desarrollado para evaluar y rehabilitar el equilibrio de pacientes hemiparéticos por accidente cerebrovascular. Método. Este es un estudio cuasiexperimental de series de tiempo en el que participaron 25 pacientes hemiparéticos post ACV. La intervención duró 10 semanas, con sesiones dos veces por semana. Se evaluaron: fuerza muscular del cuádriceps femoral (dinamometría), equilibrio (estabilometría dinámica y escala de equilibrio de Berg), movilidad funcional (Timed Up and Go Test) y percepción de la calidad de vida (perfil de salud de Nottingham). Resultados. Se observaron mejoras significativas con un gran tamaño del efecto (TDE) en todas las variables, a excepción de la fuerza muscular en la extremidad no parética, que presentó TDE moderado. Destacan los incrementos en todas las pruebas de equilibrio ( $p<0,001 ;$ TDE: 0,9 ). Conclusión. Un programa de ejercicios con JS parece ser una buena opción de tratamiento para pacientes hemiparéticos después de un ictus, especialmente para mejorar el equilibrio. Este recurso puede contribuir al proceso de rehabilitación de estos pacientes.
\end{abstract}

Palabras clave. Videojuegos; Rehabilitación; Equilibrio Postural; Hemiparesia; Accidente vascular cerebral

Trabalho realizado na Associação Catarinense de Ensino / Faculdade Guilherme Guimbala (ACE/FGG). Curso de Fisioterapia. Núcleo de Pesquisa em Neuroreabilitação (NUPEN). Joinville-SC, Brasil.

\title{
INTRODUÇÃO
}

O Acidente Vascular Cerebral (AVC) é considerado mundialmente como a segunda maior causa de morte $^{1}$ e a principal no Brasil, sendo também responsável por elevados índices de morbidades e incapacidades funcionais provisórias ou permanentes. Entre as variadas manifestações clínicas da doença, destaca-se o quadro de hemiparesia que corresponde a perda parcial de força muscular (FM) no hemicorpo contralateral ao hemisfério afetado². 
Pacientes hemiparéticos pós-AVC possuem déficits funcionais residuais, que incluem alterações do equilíbrio, especialmente pela perda do controle de tronco ${ }^{3}$. Estes pacientes apresentam frequentemente assimetria do peso corporal, deslocando o peso para o lado não parético, além de outras alterações como: instabilidades posturais, atrasos nas respostas motoras, atividade de contração muscular anormal e temporização anormal ${ }^{4}$.

O equilíbrio é fundamental para a realização de diferentes tarefas, sendo considerado um preditor para a execução das atividades de vida diárias (AVDs) ${ }^{5}$. Entende-se que a perda de equilíbrio pós-AVC gera limitações na marcha, acentuando o risco de quedas, assim, surge uma importante redução da independência funcional, que por sua vez, acarreta piora da qualidade de vida (QV) desta população6,7.

Nos últimos anos, alguns programas de reabilitação de equilíbrio para pacientes pós-AVC foram adaptados visando uma maior eficácia na recuperação funcional ${ }^{7}$. Por exemplo, tecnologias inovadoras estão sendo aplicadas, como o uso do biofeedback ${ }^{2}$, incluindo sistemas de realidade virtual $(R V)$, que fornecem um ambiente enriquecido com objetivos específicos de tarefas e práticas repetitivas ${ }^{7}$. Além das tecnologias de RV, existem os Jogos Sérios (JS), que consistem em jogos criados com um objetivo e conteúdo específico, permitem customizações e oferecem maiores possibilidades de treinamento, aprendizado e mudanças ao paciente ${ }^{8}$. 
A utilização de JS em hemiparéticos pós-AVC tem sido considerada uma estratégia promissora, visto que utiliza de forma combinada três elementos fundamentais da reabilitação: a intensidade, repetitividade e a prática orientada à tarefa 9 . Desta forma, JS favorecem o aumento da atenção e da motivação dos pacientes durante as sessões de reabilitação ${ }^{10}$. Entre os estudos realizados com hemiparéticos por AVC, a maioria são voltados para a recuperação do membro superior e, quando para membros inferiores, poucos são específicos para o equilíbrio.

Percebe-se que atualmente, ainda existe uma escassez de estudos que indiquem os efeitos de programas de reabilitação baseados em JS para melhora do equilíbrio em hemiparéticos pós-AVC. Assim, o objetivo do presente estudo foi verificar os efeitos terapêuticos de um programa de exercícios utilizando um JS desenvolvido para reabilitação do equilíbrio de pacientes hemiparéticos pós-AVC.

\section{MÉTODO}

\section{Amostra}

Trata-se de um estudo de caráter quase-experimental com delineamento do tipo séries de tempo, envolvendo 12 pacientes hemiparéticos por AVC, estáveis clinicamente, na fase crônica da doença e com faixa etária a partir de 18 anos. Como critérios de exclusão foram adotados: hemiparesia decorrente de outras patologias, pacientes hemiplégicos, comprometimento motor bilateral simétrico, comprometimento visual e/ou auditivo severo, pacientes não 
cooperativos e/ou com déficit cognitivo grave, pacientes incapazes para bipedestação de maneira independente (mesmo com a utilização de dispositivos de auxílio) e pacientes que estavam realizando, ou que tivessem realizado nos últimos 3 meses aplicação de toxina botulínica ou qualquer outro tipo de reabilitação para tronco e/ou membros inferiores.

Este estudo foi aprovado pelo Comitê de Ética em Pesquisas Envolvendo Seres Humanos da Universidade do Estado de Santa Catarina e está registrado sob o número 45881615.9.0000.0118 (CAAE).

\section{Procedimento}

Todos os participantes receberam esclarecimentos quanto aos procedimentos que seriam realizados $e$ assinaram o Termo de Consentimento Livre e Esclarecido (TCLE). Um questionário foi previamente aplicado com 0 intuito de coletar todas as informações relevantes para realização do projeto.

Após a aplicação do questionário, procederam-se as demais avaliações. Os instrumentos utilizados foram escolhidos de acordo com os domínios da Classificação Internacional de Funcionalidade, Incapacidade e Saúde $(\mathrm{CIF})^{11}$.

Em relação ao domínio Função/Estrutura Corporal $(C I F)$, foram utilizados os seguintes testes e instrumentos:

- Dinamometria para avaliação da FM do quadríceps femoral. Foi utilizado o dinamômetro digital portátil 
Chatillôn ${ }^{\circledR}$, modelo DFS II Series. Para a realização do teste, o paciente foi posicionado sentado em uma maca, com o tronco apoiado em um encosto garantindo uma estabilidade, com as pernas pendentes, quadril em $110^{\circ}$ de flexão em relação ao tronco e joelhos fletidos a $90^{\circ 12}$. O dinamômetro era sustentado pelo examinador que estava em frente ao paciente, sendo posicionado no nível do terço distal da perna (logo acima da região maleolar), na face anterior. O paciente sempre foi orientado a realizar o máximo de força possível;

- Mini Exame do Estado Mental (MEEM) para avaliar o nível cognitivo dos pacientes. Destaca-se que este instrumento foi utilizado apenas para triagem dos pacientes, sendo adotados os pontos de corte propostos por Bertolucci et al. ${ }^{13}$;

- Jogo Sério MyBalance para avaliação (estabilometria) e treinamento do equilíbrio. A prancha foi posicionada em frente a uma projeção de multimídia a uma distância de $3 \mathrm{~m}$, com o paciente em pé e descalço sobre a prancha, olhando para a projeção com o centro posicionado à altura dos olhos. Os pés ficavam afastados em uma posição natural e confortável, não ultrapassando a largura dos ombros. A Figura 1-A mostra a tela do jogo visualizada pelo paciente e a numeração das posições do alvo. Esta avaliação foi realizada por meio de duas tarefas distintas, sendo 0 tamanho do alvo configurado da mesma maneira, tamanho grande ( $5^{\circ}$ de inclinação da prancha) conforme ilustrado na Figura 1-B, para ambas as tarefas. Na primeira tarefa, chamada de Estabilometria Dinâmica Básica (EDB), o modo 
de movimentação do alvo foi definido para o modo manual e o alvo foi colocado no centro da tela, que corresponde a posição 0 da Figura $1-A$, onde permanecia fixo por 30 segundos. No segundo teste, chamado de Estabilometria Dinâmica Sequencial (EDS), o modo de movimento do alvo foi ajustado para o modo sequencial e o alvo se movia sequencialmente, da posição 0 para 8 da Figura 1-A, permanecendo 5 segundos em cada posição. O objetivo do teste era que o paciente mantivesse o seu centro de pressão (COP) dentro do alvo, quando alcançado este objetivo o alvo apresentava cor verde (Figura 1-C); quando o paciente não conseguia manter o seu COP no alvo, ele apresentava cor vermelha (Figura 1-D), dando um feedback instantâneo ao indivíduo. Para análise, foi utilizada a média do melhor escore de cada um dos dias de avaliação.

Para o domínio Atividades (CIF), foram utilizados os seguintes instrumentos:

- $\quad$ Timed Up and Go Test (TUGT) para avaliação da mobilidade funcional, por meio da mensuração do tempo de realização da tarefa proposta (paciente se levantar de uma cadeira, caminhar em linha reta por $3 \mathrm{~m}$, virar, caminhar de volta para a cadeira e sentar-se);

- Escala de Equilíbrio de Berg (EEB) para avaliar o equilíbrio funcional, que envolve a realização de 14 atividades relacionadas com as AVDs. 
Figura 1. Tela do JS MyBalance.

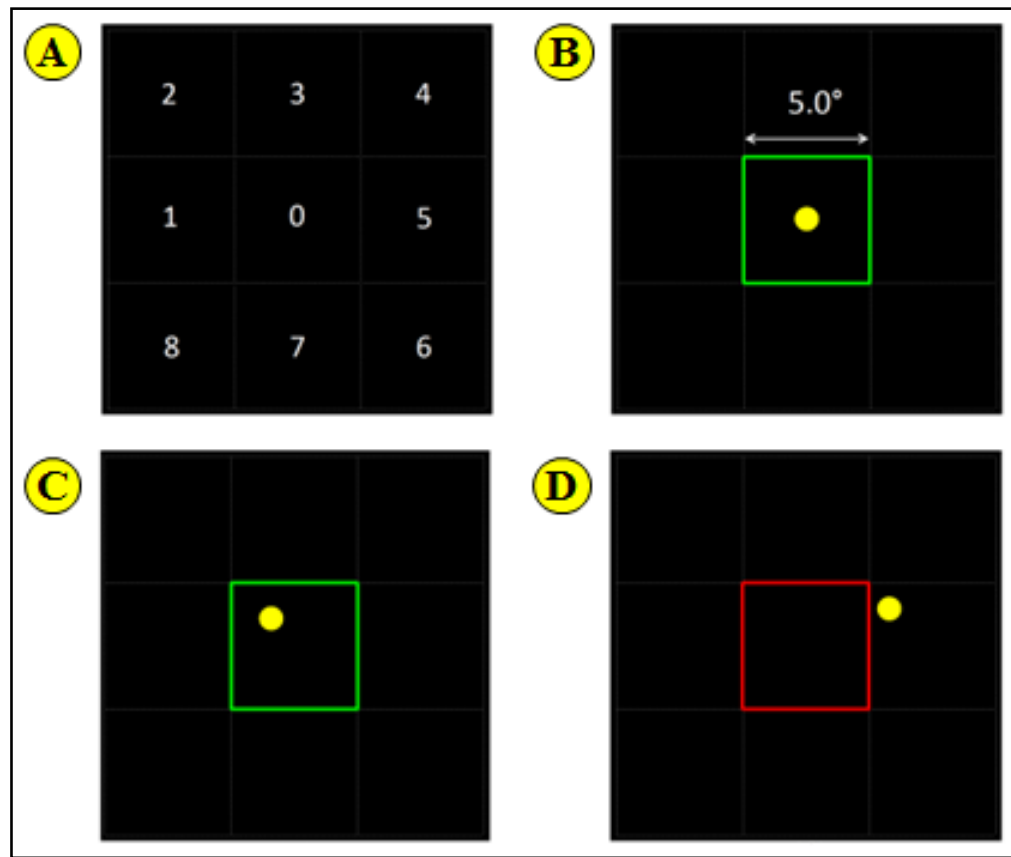

(A): numeração das posições de alvo nos modos de avaliação; $(B)$ : maior tamanho do alvo (a inclinação máxima da prancha para que a bola permaneça dentro do alvo, a partir do seu centro, é de \pm 2.5 graus); (C): feedback visual com o alvo na cor verde, indicando que a bola está dentro do alvo; (D): feedback visual com o alvo na cor vermelha, indicando que a bola está fora do alvo.

- Cada atividade é avaliada em uma escala ordinal que varia de 0 a 4 , onde 0 indica o nível mais baixo de função e 4 o nível mais alto de função. A pontuação varia de 0 a 56, quanto maior a pontuação, melhor é o controle postural.

Já para o domínio Participação (CIF), foi utilizado:

- $\quad$ Perfil de Saúde de Nottingham (PSN), para avaliar a percepção de QV dos pacientes no período pré- e pósintervenção. Os pacientes foram classificados com: baixa (26-38), moderada (13-25) ou alta (0-12) percepção da QV.

Foram realizadas três avaliações antes de iniciar o programa de reabilitação (pré-intervenção) e três avaliações ao término do programa (pós-intervenção). Todas as 
avaliações foram em dias alternados e realizadas pelos mesmos examinadores.

A sequência de avaliação foi: questionário em forma de entrevista para obtenção de todas as informações gerais relevantes para o estudo; MEEM; PSN; TUGT; EEB; dinamometria; avaliação do equilíbrio dinâmico com o JS MyBalance. Destaca-se que o questionário para obtenção de dados gerais e o MEEM foram utilizados apenas no período pré-intervenção, uma vez; as avaliações com o TUGT, EEB e dinamometria foram realizadas duas vezes no período pré- e pós-intervenção; enquanto a avaliação do equilíbrio com o JS foi realizada três vezes no período pré- e pós-intervenção.

Após a fase de avaliação os pacientes foram submetidos ao período de tratamento, que consistiu em 10 semanas consecutivas, com frequência de duas sessões semanais, totalizando 20 sessões. O protocolo de tratamento implementado foi um programa de reabilitação para o equilíbrio baseado em exercícios utilizando o JS MyBalance. Dois modos do JS foram utilizados: sequencial e aleatório. $\mathrm{O}$ modo sequencial consistia na tarefa de posicionar o COP dentro do alvo que se deslocava de forma sequencial, continuamente, da posição 0 até a posição 8 . 0 modo aleatório de treinamento exigia do paciente 0 posicionamento do COP dentro do alvo que se deslocava de forma aleatória dentre as posições 0 a 8 .

Este programa de exercícios foi dividido em duas fases com 10 sessões: Fase 1 ( 3 séries do JS em cada modo) e Fase 2 ( 4 séries do JS em cada modo). Para todos os 
pacientes foi padronizada a mesma configuração do jogo (tamanho do alvo, tamanho da bola e tempo). Em cada sessão, primeiramente foi realizada mobilização passiva, alongamentos e exercícios ativo-assistidos com o hemicorpo parético, durante um período de 10 minutos, após foi iniciado o treinamento. Cada série do jogo tinha duração de 3 minutos, com intervalo de 1 minuto entre elas para descanso. Iniciava-se sempre pelo modo sequencial, e após, eram realizados os exercícios no modo aleatório.

Destaca-se que, por precaução, antes e ao término de todos os atendimentos foram verificados os sinais vitais pressão arterial e frequência cardíaca dos pacientes. Além disso, durante a intervenção, caso o paciente relatasse malestar ou qualquer outro desconforto que pudesse trazer riscos a sua saúde, a sessão era interrompida. Felizmente, não foi constatada nenhuma ocorrência durante e após as sessões.

\section{Análise Estatística}

Os dados foram analisados por meio do software SPSSIBM, versão 20.0 for Windows. Foi utilizada estatística descritiva (média e desvio padrão) e distribuição de frequências com valores absolutos e percentuais, Teste de Shapiro-Wilk (verificação da normalidade dos dados), ANOVA One Way Relacionada, Teste $t$ de Student Pareado e Teste de Wilcoxon para soma de postos (comparação das variáveis no período pré- e pós-intervenção, para dados paramétricos e não paramétricos, respectivamente). 0 
tamanho de efeito (TDE) foi calculado para complementar a análise dos efeitos clínicos da intervenção sobre cada variável. Para classificação do TDE dos dados paramétricos, foram considerados os seguintes valores: 0,8, 0,5 e 0,2 (grande, moderado e pequeno, respectivamente). Para os dados não paramétricos foram considerados: 0,5, 0,2 e 0,1 (grande, moderado e pequeno, respectivamente) ${ }^{14}$. Foi adotado um nível de significância de $5 \%$ para todos os testes.

\section{RESULTADOS}

Foram avaliados e tratados 12 pacientes hemiparéticos por AVC (6 do sexo masculino), com idade média de 59,9 $\pm 10,2$ anos. A Tabela 1 apresenta a caracterização geral dos participantes.

Tabela 1. Caracterização geral dos pacientes.

\begin{tabular}{|c|c|c|}
\hline Características $(n=12)$ & $\overline{\mathbf{x}} \pm \mathbf{s}$ & $f(\%)$ \\
\hline \multicolumn{3}{|l|}{ Sexo } \\
\hline Feminino & --- & $6(50,0)$ \\
\hline Masculino & --- & $6(50,0)$ \\
\hline Idade (anos completos) & $59,9 \pm 10,2$ & --- \\
\hline \multicolumn{3}{|l|}{ Etnia } \\
\hline Branco & --- & $10(83,3)$ \\
\hline Negro & --- & $2(16,7)$ \\
\hline Tempo de AVC (meses) & $37,3 \pm 32,9$ & --- \\
\hline \multicolumn{3}{|l|}{ Lateralidade autorrelatada } \\
\hline Destro & --- & $12(100,0)$ \\
\hline Sinistro/Canhoto & --- & $0(0)$ \\
\hline \multicolumn{3}{|l|}{ Hemiparesia } \\
\hline Direita & --- & $9(75,0)$ \\
\hline Esquerda & --- & $3(25,0)$ \\
\hline
\end{tabular}


Todas as variáveis utilizadas na caracterização geral dos pacientes, apresentaram uma distribuição normal indicada pelo teste de Shapiro-Wilk. Destaca-se que todos os pacientes eram hemiparéticos crônicos e destros, sendo constatada uma prevalência do quadro de hemiparesia no dimídio corporal direito.

A Tabela 2 apresenta os resultados dos testes de comparação entre as medidas pré- e pós-intervenção, das variáveis FM do quadríceps femoral (parético e não parético), estabilometria dinâmica (básica e sequencial), EEB e TUGT. Todas estas variáveis já foram classificadas na metodologia segundo a CIF. Os dados relativos à FM e estabilometria pertencem ao domínio Função/Estrutura Corporal, enquanto o TUGT e a EEB pertencem ao domínio Atividades.

Tabela 2. Resultados dos testes de comparação das variáveis pertencentes aos domínios função / estrutura corporal e atividades da CIF.

\begin{tabular}{lcccc}
\hline Variável (n=12) & $\begin{array}{c}\text { Pré } \\
(\overline{\mathbf{x}} \pm \mathbf{s})\end{array}$ & $\begin{array}{c}\text { Pós } \\
(\overline{\mathbf{x}} \pm \mathbf{s})\end{array}$ & P & TDE \\
\hline $\begin{array}{l}\text { Função / } \\
\text { Estrutura corporal }\end{array}$ & & & & \\
FQFP (kgf) & $15,9 \pm 5,0$ & $17,8 \pm 5,2$ & 0,001 & 0,8 \\
FQFNP (kgf) & $22,4 \pm 4,6$ & $25,5 \pm 3,7$ & 0,018 & 0,6 \\
EDB* & $23,7 \pm 15,2$ & $67,0 \pm 22,1$ & $<0,001$ & 0,9 \\
EDS* & $13,1 \pm 10,1$ & $38,9 \pm 13,8$ & $<0,001$ & 0,9 \\
Atividades & & & & 0,8 \\
TUGT (s) & $26,1 \pm 12,9$ & $22,5 \pm 11,2$ & $<0,001$ & 0,9 \\
EEB & $43,5 \pm 6,5$ & $49,9 \pm 4,5$ & $<0,001$ & 0 \\
\hline TDE: tamanho de efeito; FQFP: forca do quadríceps femoral parético; FQFNP: força do quadríceps femoral
\end{tabular}

TDE: tamanho de efeito; FQFP: força do quadríceps femoral parético; FQFNP: força do quadríceps femoral não parético; EDB*: escore obtido com a estabilometria dinâmica básica (modo manual) no JS MyBalance; EDS: escore obtido com a estabilometria dinâmica sequencial (modo sequencial) no JS MyBalance; TUGT: timed up and go test; EEB: escala de equilíbrio de Berg; n: amostra total. 
Merece destaque o fato de que foram obtidas melhoras significativas e com grande TDE em todas as variáveis, com exceção da FM no quadríceps femoral não parético. O equilíbrio dinâmico foi a variável que apresentou maiores incrementos.

A Tabela 3 resume os resultados dos testes de comparação entre as medidas pré- e pós-intervenção, da variável obtida com o PSN, que pertence ao domínio Participação. Foram encontradas melhoras significativas e com grande TDE nesta variável.

Tabela 3. Resultados do teste de comparação da variável pertencente ao domínio participação da CIF.

\begin{tabular}{lcccc}
\hline PSN (n=12) & $\begin{array}{c}\text { Pré } \\
\mathbf{f ( \% )}\end{array}$ & $\begin{array}{c}\text { Pós } \\
\mathbf{f ( \% )}\end{array}$ & P & TDE \\
\hline Baixa percepção & $1(8,3)$ & $0(0)$ & \\
Moderada percepção & $6(50,0)$ & $4(33,3)$ & 0,010 & 0,5 \\
Alta percepção & $5(41,7)$ & $8(66,7)$ & & \\
\hline PSN: perfil de saúde de Nottingham; TDE: tamanho de efeito; n: amostra total.
\end{tabular}

PSN: perfil de saúde de Nottingham; TDE: tamanho de efeito; n: amostra total.

\section{DISCUSSÃO}

As sequelas motoras resultantes de um AVC englobam alterações do equilíbrio, as quais impactam de forma importante na independência funcional, resultando em piora da QV dos pacientes. ${ }^{6}$. Por este motivo, buscam-se estratégias de tratamento eficazes para este tipo de disfunção. O uso de tecnologias de RV e jogos na reabilitação pós-AVC tem aumentado ${ }^{15}$, entre estes recursos destacamse os JS, capazes de aumentar a demanda atencional e a motivação durante o tratamento, além de permitirem a 
customização de acordo com as limitações apresentadas pelo paciente ${ }^{10,16}$. Já existem evidências de que o treinamento de equilíbrio com JS resulta em melhoras para os pacientes ${ }^{8}$.

No presente estudo, o programa de exercícios com o JS MyBalance resultou em melhoras significativas e com grande TDE em todas as variáveis investigadas, com exceção apenas da FM do quadríceps femoral no membro não parético que, embora significativas, apresentaram um TDE moderado.

A fraqueza muscular consiste no comprometimento primário pós-AVC ${ }^{17}$. A redução dos níveis desta variável impacta na independência funcional, à medida que limita a realização das AVDs, estando associada também ao aumento do risco de quedas nestes pacientes ${ }^{18}$. Assim, restaurar a FM tem sido uma meta importante da reabilitação do equilíbrio e da marcha, visto que está relacionada com uma distribuição de peso mais simétrica e com a capacidade de deslocamento de peso ${ }^{2,19}$. Com relação ao efeito da intervenção sobre esta variável, destaca-se que foram obtidos ganhos significativos para QF bilateralmente, com grande $\operatorname{TDE}(0,8)$ no lado parético e moderado $(0,6)$ no não parético. Vindo ao encontro destes achados, um experimento realizado com 40 hemiparéticos por AVC, avaliou os efeitos do treinamento da marcha assistida por robô utilizando RV e estimulação auditiva sobre o equilíbrio e a marcha ${ }^{20}$. Foram constatadas melhoras na capacidade de equilíbrio e da marcha, superiores no grupo que utilizou a RV, sendo estas associadas com um incremento da FM dos membros inferiores, especialmente no lado parético. Acredita-se que, 
no presente estudo, a melhora superior da FM evidenciada no lado parético, se dá pelo fato do treinamento exigir do paciente maior descarga de peso neste membro, o que normalmente não acontece no cotidiano. Portanto, as exigências do JS em uma plataforma instável associadas ao comando verbal realizado pelo fisioterapeuta, estimulam uma redistribuição de peso mais adequada nos membros inferiores. Estudo sobre treinamento por meio do Nitendo Wii Fit $^{\mathrm{TM}}$ com hemiparéticos por AVC, em uma prancha de equilíbrio instável, mostrou incrementos na FM do membro parético e na descarga de peso neste membro ${ }^{19}$. A presença de uma tarefa desafiadora auxilia na obtenção de ganhos, estando relacionada à ativação dos vários sistemas responsáveis pelo equilíbrio.

Devido aos deficits funcionais residuais do AVC, assimetrias na descarga de peso corporal, prejuízos no controle de tronco, entre outras alterações são frequentes, contribuindo para a existência de déficits do equilíbrio ${ }^{3,4}$. 0 equilíbrio é de fundamental importância para o ser humano, pois está diretamente relacionado à marcha, que é uma das principais habilidades motoras existentes ${ }^{6}$. Os exercícios realizados com o JS promoveram melhoras e com grande TDE nesta variável $(p<0,001 ; \operatorname{TDE}=0,9)$, tanto no teste de EDB (modo manual), quanto na EDS (modo sequencial). Um estudo realizado com 18 pacientes em fase crônica do AVC, utilizando o mesmo JS MyBalance, mostrou que após o programa de treinamento, os pacientes conseguiram atingir 
níveis de equilíbrio satisfatórios, muito semelhantes ao de pessoas saudáveis utilizadas como controle ${ }^{8}$.

Com o intuito de avaliar o equilíbrio no ambiente clínico e em pesquisas foi desenvolvida a EEB, que possui boa confiabilidade em relação à monitorização do equilíbrio, prognóstico da disfunção, predição do risco de quedas, triagem de pacientes para o processo de reabilitação e avaliação das respostas ao tratamento ${ }^{21}$. Os resultados obtidos com o treinamento baseado no JS indicam melhoras com grande TDE ( $p<0,001 ;$ TDE 0,9$)$ nesta variável. Uma revisão sistemática abordou a utilização do Nitendo ${ }^{\circledR}$ Wii como recurso de tratamento, onde a maioria dos indivíduos eram hemiparéticos por $\mathrm{AVC}^{21}$. Os resultados desta revisão vêm ao encontro dos nossos, pois foi verificada a eficácia deste tipo de intervenção sobre o equilíbrio e a FM.

Um ensaio clínico randomizado com 25 hemiparéticos pós-AVC, utilizou um programa de treinamento baseado em jogos digitais associados com um treinamento cognitivo ${ }^{22}$. Os resultados foram positivos, apontando melhora do equilíbrio, além da mobilidade funcional e de funções cognitivas. Outro ensaio clínico randomizado com 60 pacientes mostrou que, após a intervenção, as médias foram superiores na EEB para os pacientes que realizaram treinamento utilizando o biofeedback quando comparados ao grupo que realizou exercícios convencionais ${ }^{2}$. Tal achado reforça a importância da utilização deste recurso, que é tido como favorável para a melhora da situação motora dos pacientes pós-AVC. O JS MyBalance utiliza o recurso de 
biofeedback de forma incorporada, o que potencializa ainda mais o seu uso na reabilitação.

O TUGT é uma medida clínica objetiva, válida e confiável para avaliar a mobilidade e o equilíbrio funcional, sendo considerada preditiva para o risco de quedas ${ }^{23}$. Nesta variável, foram constatadas melhoras com grande $\operatorname{TDE}(0,8)$ após a intervenção com o JS. Uma revisão sistemática com meta-análise de 21 estudos sobre o efeito do treinamento com RV no equilíbrio e na capacidade de marcha de pacientes pós-AVC ${ }^{24}$, concluiu que o treinamento baseado em RV é capaz de promover melhoras em variáveis relacionadas à mobilidade funcional. Na mesma linha, outra meta-análise sobre os efeitos da RV analisou 21 estudos, que envolveram um total de 562 pacientes, também evidenciando melhoras da mobilidade funcional, além da FM de membros inferiores, do equilíbrio e de parâmetros da marcha ${ }^{25}$.

No passado, a prática da reabilitação era direcionada para os deficits físicos apresentados pelo paciente. Atualmente, existe uma maior atenção em aumentar a participação destes indivíduos em AVDs, com o intuito de maximizar a QV, que é influenciada na medida que o AVC compromete a autoestima, além da interação com a família e com a sociedade ${ }^{26}$. A QV corresponde à maneira como as pessoas se sentem ou se percebem, com relação as suas condições de saúde ${ }^{27}$. A utilização do JS resultou em melhoras significativas e com grande $\operatorname{TDE}(0,5)$ na variável percepção da QV. Tais achados são corroborados por uma revisão integrativa sobre a reabilitação de pacientes pós-AVC 
com a utilização de jogos comerciais que envolviam RV 27 . Após análise final de 13 artigos, os autores concluíram que o tratamento baseado na associação da RV com a fisioterapia convencional resulta em efeitos positivos sobre a QV. Porém, é importante e válido destacar que a QV se trata de uma variável multifatorial, podendo ser influenciada por vários elementos distintos ${ }^{28}$, não permitindo afirmar de forma conclusiva que o efeito de uma intervenção é o único responsável pelos achados nesta variável.

Frequentemente indivíduos que sofreram um AVC passam por um processo de reabilitação que é longo e cansativo, surgindo problemas de motivação e adesão do paciente, resultando até mesmo em abandono do tratamento ${ }^{29}$. Os JS são recursos capazes de reduzir estes fatores negativos, uma vez que modificam a forma da reabilitação, tornando o paciente mais ativo no processo, aumentando a demanda atencional e a motivação dele, além de promover maior prazer na execução do treinamento ${ }^{30}$.

\section{CONCLUSÃO}

A análise dos efeitos terapêuticos obtidos com 0 programa de exercícios baseado no JS MyBalance, indica melhoras significativas em todas as variáveis estudadas. Estes achados apontam que os JS podem ser considerados como um recurso potencialmente benéfico para a reabilitação de pacientes hemiparéticos por AVC.

Devido à especificidade do treinamento, utilizando uma plataforma instável para trabalhar o equilíbrio, ficou evidente 
o efeito obtido com a intervenção baseada no JS, principalmente nos testes de estabilometria e na EEB. Melhoras nestas variáveis resultam em ganhos em outras atividades cotidianas como a marcha, aumentando a independência funcional e impactando desta forma na QV dos pacientes.

\section{REFERÊNCIAS}

1.Benjamin EJ, Blaha MJ, Chiuve SE, Cushman M, Das SR, Deo R, et al. Heart disease and stroke statistics-2017 Update: a report from the American Heart Association. Circulation 2017;135:146-603. http://dx.doi.org/10.1161/CIR.0000000000000485

2.Najafi Z, Rezaeitalab F, Yaghubi M, Manzari ZS. The effect of biofeedback on the motor - muscular situation in rehabilitation of stroke patients: a randomized controlled trial. Int J Caring Sci 2018;7:89-93. http://dx.doi.org/10.15171/jcs.2018.014

3. Caires TA, Silva GV, Castro SS, Souza LAPS. Trunk control and its relation with clinical condition, central anatomic area and postcerebrovascular accident phase. Fisioter Pesq 2018;25:224-8. http://dx.doi.org/10.1590/1809-2950/17025025022018

4.Annethattil A, Paul J, Kumar P, Krishnamurthy R, Kumar A. The effectiveness of balance training without visual feedback in stroke subjects. IJMAES 2018;4:448-52.

http://dx.doi.org/10.36678/ijmaes.2018.v04i01.002

5.Van Duijnhoven HJ, Heeren A, Peters MA, Veerbeek JM, Kwakkel G, Geurts AC, et al. Effects of exercise therapy on balance capacity in chronic stroke: systematic review and meta-analysis. Stroke 2016;47:2603-10.

http://dx.doi.org/10.1161/STROKEAHA.116.013839

6.Song M, Lee J, Shin W. Minimal clinicaly important difference of berg balance scale scores in people with acute stroke. Phys Ther Rehabil Sci 2018;7:102-8. http://dx.doi.org/10.14474/ptrs.2018.7.3.102

7.Yatar GI, Yildiri, SA. Wii fit balance training or progressive balance training in patients with chronic stroke: a randomized controlled trial. J Phys Ther Sci 2015;27:1145-51.

http://dx.doi.org/10.1589/jpts.27.1145

8. Noveletto F, Soares AV, Mello BA, Savegnani CN, Eichinger FLF, Hounsell MS, et al. Biomedical serious game system for balance rehabilitation of hemiparetic stroke pacients. IEEE Trans Neural Syst Rehab Eng 2018;26:2179-88.

http://dx.doi.org/10.1109/TNSRE.2018.2876670

9. Noveletto F, Soares AV, Eichinger FLF, Domenech SC, Hounsell MS, Bertemes Filho P. Biomedical serious game system for lower limb motor 
rehabilitation of hemiparetic stroke patients. IEEE Trans Neural Syst Rehab Eng 2020;28:1481-7.

http://dx.doi.org/10.1109/TNSRE. 2020.2988362

10.Juras G, Brachman A, Michalska J, Kamieniarz A, Pawlowski M, Hadamus $A$, et al. Standards of virtual reality application in balance training programs in clinical practice: a systematic review. Games Health J 2019;8:101-11. http://dx.doi.org/10.1089/g4h.2018.0034 11. Who - World Health Organization. Classificação internacional de funcionalidade, incapacidade e saúde - CIF. 2004;238p. https://catalogo.inr.pt/documents/11257/0/CIF+2004

12. Cooper A, Alghamdi GA, Alghamdi MA, Altowaijri A, Richardson R. The relationship of lower limb muscle strength and knee joint hyperextension during the stance phase of gait in hemiparetic stroke patients. Physiother Res Int 2011;17:150-6.

http://dx.doi.org/10.1002/pri.528

13. Bertolucci PHF, Brucki SMD, Campacci SR, Juliano YO. Mini-exame do estado mental em uma população geral: impacto da escolaridade. Arq Neuropsiquiatr 1994;52:1-7. http://dx.doi.org/10.1590/S0004282X1994000100001

14.Cohen J. Statistical power analysis for the behavioral sciences. 2nd edition. New Jersey: Lawrence Erlbaum Associates (Pub); 1988. http://www.utstat.toronto.edu/ brunner/oldclass/378f16/readings/C ohenPower.pdf

15. Winstein CJ, Stein J, Arena R, Bates B, Cherney LR, Cramer SC, et al. Guidelines for adult stroke rehabilitation and recovery. Stroke 2016;47:98-169. http://dx.doi.org/10.1161/STR.0000000000000098 16. Dias P, Silva R, Amorim P, Lains J, Roque E, Pereira ISF, et al. Using virtual reality to increase motivation in poststroke rehabilitation. IEEE Comput Graph Appl 2019;39:64-70.

http://dx.doi.org/10.1109/MCG.2018.2875630

17. Dorsch S, Ada L, Canning CG. Lower limb strength is significantly impaired in all muscle groups in ambulatory people with chronic stroke: a cross-sectional study. Arch Phys Med Rehabil 2016;97:522-7. http://dx.doi.org/10.1016/j.apmr.2015.10.106

18.Franciulli PM, Magaldi CM, Bigongiari A, Barbanera M. Efeito do treinamento resistido em hemiparéticos crônicos no equilíbrio e torque isocinético do joelho. Rev Bras Cienc Saúde 2018;22:125-30. http://dx.doi.org/10.22478/ufpb.2317-6032.2018v22n2.32752

19.Miranda CS, Oliveira TP, Gouvêa JXM, Perez DB, Marques AP, Piemonte MP. Balance training in virtual reality promotes performance improvement but not transfer to postural control in people with chronic stroke. Games Health J 2019;8:294-300.

http://dx.doi.org/10.1089/g4h.2018.0075

20.Park J, Chung Y. the effects of robot-assisted gait training using virtual reality and auditory stimulation on balance and gait abilities in persons with stroke. NeuroRehab 2018;43:227-35. http://dx.doi.org/10.3233/NRE-172415

21.Lopes PC, Carvalho JDP, Neto HBDS, Souza KCLD, Taddeo PDS, Praça $L R$, et al. Realidade virtual em uma estratégia de reabilitação 
neurofuncional: revisão sistemática. Varia Sci Cienc Saúde 2017;3:113. https://dx.doi.org/10.48075/vscs.v3i1.16813

22.Kannan L, Vora J, Bhatt T, Hughes S. Cognitive-motor exergaming for reducing fall risk in people with chronic stroke: a randomized controlled trial. NeuroRehab 2019;44:493-510.

http://dx.doi.org/10.3233/NRE-182683

23.Chan PPK, Si Tou JIF, Tse MMY, Ng SSM. The reliability and validity of the timed up and go (motor) test for people with chronic stroke. Arch Phys Med Rehabil 2017;98:2213-20.

http://dx.doi.org/10.1016/j.apmr.2017.03.008

24.De Rooij IJM, Van De Port IGL, Meijer JWG. Effect of virtual reality training on balance and gait ability in patients with stroke: systematic review and meta-analysis. Phys Ther 2016;96:1905-18.

http://dx.doi.org/10.2522/ptj.20160054

25. Lee HS, Park YJ, Park SW. The effects of virtual reality training on function in chronic stroke patients: a systematic review and metaanalysis. BioMed Res Int 2019;8:1-12.

http://dx.doi.org/10.1155/2019/7595639

26. Lapadatu I, Morris R. The relationship between stroke survivors' perceived identity and mood, self-esteem and quality of life. Neuropsychol Rehabil 2019;29:199-213.

http://dx.doi.org/10.1080/09602011.2016.1272468

27.Aramaki AL, Sampaio RF, Reis ACS, Cavalcanti A, Dutra FCS. Virtual Reality In the rehabilitation of patients with stroke: an integrative review. Arq Neuropsiquiatr 2019;77:268-78.

http://dx.doi.org/10.1590/0004-282X20190025

28. Tastekin N. Rehabilitation and quality of life in stroke patients. Turk J Phys Med Rehabil 2015;61:1-8.

http://dx.doi.org/10.5152/tftrd.2015.001

29.Mcgrane N, Galvin R, Cusack T, Stokes E. Addition of motivational interventions to exercise and traditional physiotherapy: a review and meta-analysis. Physiotherapy 2015;101:1-12.

http://dx.doi.org/10.1016/j.physio.2014.04.009

30.Soares AV, Borges-Júnior NG, Hounsell MS, Marcelino E, Rossito GM, Sagawa-Júnior Y. A serious game developed for physical rehabilitation of frail elderly. Eur Res Telemed 2016;45-53. http://dx.doi.org/10.1016/j.eurtel.2016.05.003 